\title{
Arbor
}

\section{Cambios en los roles familiares y domésticos*}

\section{Inés Alberdi}

Arbor CLXXVIII, 702 (Junio 2004), 231-261 pp.

Los cambios en los modelos de familia se pueden considerar como un fenómeno complejo con múltiples dimensiones. Las formulas alternativas de convivencia van tomando legitimidad e incluso cobran los rasgos de un mayor compromiso emocional. Las razones que impulsan las uniones matrimoniales han cambiado. Una de las conclusiones más notables del estudio es la desaparición del concepto sacralizado del matrimonio y su sustitución por una idea más instrumental del mismo.

Una de las claves del cambio es la transformación del escenario que tienen ante sí las parejas, pasando de un esquema único de convivencia, el matrimonio, a contemplar un panorama de múltiples opciones, por las que optan en función de los estilos de vida, creencias e intereses de cada uno o según los periodos o las etapas de su vida. Sentirse libres ante el propio proyecto de vida es la filosofía que define la vida de la mayoría de la población joven española, es decir la que marca las tendencias de las futuras generaciones, y ello se refleja en la encuesta realizada en el año 2000 sobre estilos de vida familiar.

En el marco de este proceso de flexibilidad y diversidad de formas de convivencia, la encuesta refleja una creciente tolerancia hacia las parejas homosexuales; incluso respecto a encuestas anteriores se avanza en el sentido de aceptar que estas puedan unirse en matrimonio. Hay que hacer notar también como la adopción genera actitudes muy positivas sin que a ello sea obstáculo la nacionalidad de los niños adoptados. Las actitudes de solidaridad e identificación con el fenómeno de la adopción son unánimes.

* Esta conferencia presenta resultados de la Encuesta sobre «Flexibilidad y estilos de vida familiar» realizada en el año 2000 por encargo del Ministerio de Trabajo y Asuntos Sociales. 
El concepto de vida completa, de logro o éxito en la vida, ya no pasa necesariamente por contraer matrimonio aunque incluye, como ingredientes fundamentales, tener una pareja y tener un trabajo.

La decisión de tener hijos, del momento de tenerlos y el número de ellos es consecuencia de un nuevo concepto de ser padres. Nadie quiere renunciar a tener hijos en el momento actual. La maternidad sigue siendo un valor muy importante y la paternidad también. El tener un hijo entra dentro del proyecto vital de la mayoría de los jóvenes, pero está rodeado de múltiples condicionantes que hacen emerger contradicciones entre los deseos y realidades.

A través del estudio realizado aparecen de forma constante las contradicciones entre expectativas y realidades; lo que no es de extrañar en la dinámica de una sociedad sometida a importantes procesos de cambio. Esta distancia entre expectativas y realidades se agudiza en dos cuestiones, la decisión sobre el número de hijos y la actividad laboral femenina.

La tasa de fecundidad actual en España está por debajo del número ideal de hijos que desean las parejas. Tener un solo hijo, opción muy frecuente, parece una solución para cumplir con el deseo de maternidad o paternidad y a la vez cargarse lo menos posible de responsabilidades familiares. El ideal de familia incluye más hijos pero el tener sólo uno es una solución de compromiso que permite mantener otras exigencias.

El reparto del trabajo remunerado y del trabajo doméstico entre hombres y mujeres es el caballo de batalla que presentan las mujeres y se ve como condición indispensable para el logro de la familia igualitaria. Este modelo de familia igualitaria también es el preferido por los hombres, que lo incluyen dentro de sus aspiraciones; aunque no lo hagan realidad.

En esta cuestión del reparto de las tareas domésticas el estudio demuestra que existe aún una gran contradicción y una importante distancia entre las expectativas y la realidad. El ideal de familia igualitaria es mayoritario. Sin embargo en la realidad cotidiana de las familias españolas hay una gran cantidad de mujeres que no son activas y se produce, mayoritariamente, un reparto desigual de tareas y responsabilidades entre el hombre y la mujer. Las mujeres quieren tener trabajo remunerado y demandan que los hombres se incorporen de forma paritaria al trabajo familiar y doméstico.

La desigualdad en el reparto de responsabilidades domésticas, que no se comparten en la medida que deberían en una sociedad avanzada que reclama una posición de igualdad para las mujeres, es el principal obstáculo que tienen las mujeres jóvenes para poder incorporarse al empleo y progresar profesionalmente. 
El modelo igualitario, que mayoritariamente se prefiere, supone que tanto el hombre como la mujer trabajen fuera de casa y que ambos contribuyan por igual al trabajo doméstico y al cuidado familiar. Este tipo de familia igualitaria es minoritario, y se da en mayor medida en las familias dónde la mujer tiene un alto nivel educativo. En la presente fase de transición, las mujeres siguen enfrentándose a trabas en el mercado laboral. Esto implica a menudo frustración de sus expectativas y sacrificios personales. Los hombres jóvenes apoyan el cambio hacia la igualdad, pero esto no se traduce significativamente en un compromiso constante por hacerse cargo de la mitad del trabajo doméstico y familiar. El papel del hombre como principal proveedor económico de la familia contribuye a que su empleo prime en importancia sobre el empleo de la mujer.

En nuestro estudio también se han abordado las estrategias de conciliación, por ser uno de los temas que acapara actualmente el mayor interés de los estudios sobre la familia. Se trata primordialmente de saber cómo hacer compatibles la variedad de responsabilidades y tareas familiares de los adultos jóvenes, sobre todo de las mujeres que pretenden trabajar y tener hijos.

El compatibilizar maternidad y empleo es el núcleo duro de las estrategias de conciliación. Entre las dos opciones, muchas mujeres se inclinan hacia el trabajo y hacen que se resienta el proyecto familiar. He aquí según este estudio una de las razones que pueden estar influyendo en la reducción de la natalidad: tener menos hijos es una consecuencia de las estrategias para hacer compatibles el trabajo y la familia.

Como resultado de la doble carga que siguen llevando las mujeres sus carreras profesionales se ven mermadas porque tienen una sobrecarga de responsabilidades cotidianas. Y una de las consecuencias de este problema es el descenso de la natalidad. El número de hijos se reduce debido a las dificultades que encuentran las madres activas cuando tienen su primer hijo y el peligro que supondría para sus empleos y sus carreras el volver a tener otro. La reducción del número de hijos es la principal estrategia de compatibilidad entre la vida laboral y la vida familiar que están ensayando las mujeres españolas.

Reducir el número de hijos se vislumbra como la salida más coherente con la realidad de una sociedad que reconoce el valor del trabajo para las mujeres y las prepara para ello, permitiendo el acceso de un número elevado de mujeres a estudios superiores. No obstante, a pesar de la adquisición de un capital educativo, buena parte de ellas no llegan a poder invertirlo en un trabajo adecuado a sus aspiraciones y ello es un motivo de frustración que encuentra difícil solución. Las responsabilidades familiares siguen siendo la principal barrera en el mundo laboral. 
Se refleja en el estudio que apenas existen otras alternativas para las mujeres que la precariedad en el trabajo o la precariedad en la maternidad, si no se aportan otras soluciones que partan de los servicios públicos al alcance de todos y de un reparto equitativo en el interior de las familias. Optar por el trabajo a tiempo parcial, es otra fórmula que mayoritariamente se reconoce como una vía importante, realista y razonable, para salir del conflicto que enfrenta maternidad y empleo. En este sentido, llama la atención como se tiene asumido que, en todo lo que concierne a la conciliación de vida familiar y empleo, el sujeto que ha de «conciliar» siempre sea la mujer y no se piensa en un mayor equilibrio entre ambos miembros de la pareja. Solo recientemente se comienza a hablar de conciliar trabajo y familia refiriéndose tanto para los hombres como para las mujeres

Puesto que la tasa de actividad de las mujeres en edad reproductiva es una realidad positiva e irrevocable, la necesidad de encontrar respuestas sociales e institucionales al problema de la incompatibilidad entre familia y trabajo es de gran importancia. Hay que atender las necesidades de las familias desde diversos frentes: equiparación real de las oportunidades laborales de las mujeres, flexibilización de horarios de trabajo y aumento del trabajo a media jornada con derechos sociales y garantías de estabilidad, sensibilización de la sociedad de los efectos negativos de los estereotipos sexuales, y aumento de las ayudas y los servicios sociales de atención a la infancia y a la dependencia por parte del Estado.

\section{Tipos de unión familiar}

Los resultados de la encuesta reflejan que las imágenes de la familia están cambiando en la sociedad española, sobre todo entre los grupos de edad más jóvenes. La encuesta refleja que los españoles tienen unas ideas acerca del matrimonio, de las relaciones familiares, de la dedicación laboral de las mujeres, así como de las relaciones entre las mujeres y los hombres y sus hijos un tanto diferentes de las que reflejan estudios realizados con anterioridad.

En esta encuesta del año 2000 se refleja la pluralidad de opciones que están apareciendo en la sociedad española respecto del modelo que familia que cada cual asume y configura. Aunque la forma predominante de hogar familiar es la de un matrimonio que tiene hijos y convive con ellos, ha desaparecido la idea de que este tipo de familia es el único posible y 
van surgiendo, de forma numerosa en cuanto a opiniones y de forma reducida en cuanto a situaciones reales, las parejas que conviven sin casarse, las cuales incluso tienen hijos, los matrimonios que deciden no tener hijos y otras formas de convivencia entre gentes de diferentes edades, ya sean del mismo o de distinto sexo.

Se vislumbra la posibilidad de que los individuos y las parejas, de forma sucesiva, pasen por formas diferentes de convivencia. A la vez, se atenúa la idea de estabilidad y se acrecienta la desinstitucionalización. El rechazo o la posposición del matrimonio legal, y la libertad de cambiar de situación, en cuanto existe la posibilidad de divorcio o ruptura de pareja, hacen que las formas de convivencia que se establecen vayan perdiendo ese carácter de inamovilidad que tuvieron en épocas pasadas el matrimonio y la familia. Se advierte cada vez más, por ahora mayormente en términos de concepción teórica que de realidad fáctica, una cierta flexibilidad en cuanto a las formas de pareja y de convivencia, que han perdido su carácter de definitivas. Las diferentes formas de convivencia han dejado de ser opciones únicas en la vida de cada individuo y se empiezan a ver como parte de un proceso a través del cual se desarrolla la biografía individual. Van desapareciendo las reglas, o se hacen más flexibles, y las parejas se configuran a la carta, con hijos, sin hijos, viviendo con ellos o separadamente ambos progenitores, con matrimonio o sólo mediante acuerdo mutuo. Las familias que se forman son resultado de estas nuevas tendencias, derivadas del aumento del individualismo y de la libertad y, a la vez, conviven con creencias y normas del pasado que no han desaparecido aunque se han flexibilizado enormemente.

Este proceso de inercia y superación, a la vez, de las ideas del pasado se refleja muy bien en las opiniones de los diferentes grupos de edad sobre sus preferencias acerca de las formas de convivencia y las formas de celebración del matrimonio o de iniciación de la convivencia. Esta cuestión se incluía en una encuesta del Centro de Investigaciones Sociológicas dedicada a la Juventud y el Entorno Familiar (CIS 1997). Preguntando las opiniones acerca de cual era, en su opinión, la forma más adecuada de establecer la convivencia para una pareja estable, un $24 \%$ de los encuestados respondía "vivir juntos y luego casarse por la iglesia» manifestando con ello una contradicción intrínseca en sus ideas y sus preferencias. Esta opción era señalada por uno de cada cuatro jóvenes entrevistados (muestra de jóvenes entre 15 y 29 años) reflejando la coexistencia de formas tradicionales e innovadoras de pensar acerca de la pareja y del matrimonio o, dicho de otro modo, la existencia de valores contradictorios que presionan sobre los jóvenes en los momentos de defi- 
nir los modelos familiares de su preferencia. Por una parte, convivir sin matrimonio, aunque sea temporalmente, supone una posición de ruptura con los esquemas tradicionales en los que el matrimonio se consideraba requisito necesario, legal y socialmente, para la iniciación de la convivencia. A la vez, optar por el matrimonio religioso supone un cierto compromiso con los principios más tradicionales de la Iglesia católica. La Iglesia da al matrimonio el rango de sacramento, ya no solo de contrato civil, sino que lo hace requisito imprescindible y necesario para la convivencia, dictaminando como pecado la convivencia entre todos aquellos que no lo hayan contraído, sin aceptar excusas ni atenuantes. (Cuadro 1)

CUADRo 1. Formas de convivencia y formas de iniciación de la misma preferidas por los jóvenes

\begin{tabular}{|l|c|}
\hline \multicolumn{1}{|c|}{ Forma de Convivencia } & \% $\mathbf{1 9 9 7}$ \\
\hline Casarse por la iglesia & 36 \\
\hline Casarse por lo civil & 6 \\
\hline Vivir juntos y luego casarse por la iglesia & 24 \\
\hline Vivir juntos y luego casarse por lo civil & 11 \\
\hline Vivir juntos sin casarse & 18 \\
\hline No vivir juntos & 1 \\
\hline Otras respuestas, N.S/N.C & 4 \\
\hline
\end{tabular}

Fuente: «Juventud y entorno Familiar» CIS Estudio 22621997.

Podemos concluir que una parte importante de la juventud se muestra partidaria de la convivencia sin matrimonio. De hecho, sumando los partidarios de usarla temporalmente y los partidarios de verla como fórmula definitiva, son mayoría los jóvenes que ven con simpatía la convivencia sin matrimonio.

Sin embargo, al mismo tiempo, una buena parte de ellos no renuncian, a pesar de defender la pareja de hecho, al proyecto de casarse por la iglesia. Y lo más asombroso de todo es que la Iglesia católica, al menos en España, transige con esta situación y no pone el menor impedimento, ni tan siquiera algún tipo de condicionante, a las parejas que acuden a celebrar en ella su matrimonio, aún sabiendo que, de hecho, en sus formas de vida y en sus opiniones están en contradicción con sus doctrinas y sus enseñanzas. Al igual que en muchas otras cuestiones, se manifies- 


\section{Cambios en los roles familiares y domésticos}

ta en esto la importancia en nuestro país del llamado catolicismo cultural. En la sociedad española coexiste el respeto de las tradiciones y los ritos religiosos con el declive de la influencia doctrinal de la Iglesia.

Pero no es este el tema que nos interesa ahora. Al señalar esta cuestión de las celebraciones del matrimonio y de las preferencias de los jóvenes acerca de las formas de convivencia, lo que nos interesa resaltar es la diversidad y la flexibilidad que se van introduciendo en la sociedad española y que se reflejan de forma significativa en la coexistencia de valores y de comportamientos tradicionales con ideas y comportamientos que podemos considerar nuevos y rupturistas.

\section{Convivencia sin matrimonio}

El matrimonio sigue siendo una institución muy importante en la sociedad española. Sin embargo, ha perdido la hegemonía que tenía como única puerta de acceso a la formación de la familia. Los comportamientos de la población española reflejan la importancia del matrimonio, la mayoría de las familias españolas se construyen a partir del matrimonio, pero es interesante señalar los cambios que se están produciendo en las actitudes respecto de la institución matrimonial.

La convivencia sin matrimonio se acepta cada vez más por parte de la población española. Comparando las opiniones expresadas en encuestas realizadas a lo largo de la última década se advierte que, aunque son mayoría los que prefieren el matrimonio, es significativo el aumento de los que aceptan la convivencia, ya sea como alternativa al matrimonio o como periodo previo al mismo. (Cuadro 2)

CuAdro 2. Evolución de las opiniones acerca del matrimonio y de la convivencia Porcentaje de los que responden a la pregunta ¿Cuál de las siguientes formas de convivencia le parece mejor?

\begin{tabular}{|l|c|c|c|c|c|}
\hline Formas de convivencia & $\mathbf{1 9 9 2}$ & $\mathbf{1 9 9 4}$ & $\mathbf{1 9 9 5}$ & $\mathbf{1 9 9 7}$ & $\mathbf{1 9 9 8}$ \\
\hline Casarse & $77 \%$ & 68.5 & 69 & 60 & 63 \\
\hline Convivir antes de casarse & 11 & 14 & 18.5 & 17 & 17 \\
\hline Convivir sin matrimonio & 6 & 10 & 9.5 & 13.5 & 11 \\
\hline N.S/N.C / Otras & 6 & 7.5 & 3 & 9.5 & 9 \\
\hline
\end{tabular}

Fuente: Centro de Investigaciones Sociológicas CIS, Estudios 2486, 2487, 2492, 2495, 2497 
Podemos ver que han cambiado las actitudes con respecto al matrimonio y a la convivencia. De las opiniones expresadas en nuestra encuesta se desprende que son mayoría los que creen que no es importante casarse cuando se quieren tener hijos y que son mayoritarias las expresiones de comprensión y tolerancia acerca de las parejas estables que conviven sin matrimonio. (Cuadro 3)

Sin embargo los comportamientos no acompañan estas opiniones. Los comportamientos se mantienen más tradicionales y la llegada de los hijos se pospone en la mayoría de los casos a la celebración del matrimonio. Respecto de la relación entre el matrimonio y la decisión de tener hijos, las actitudes que se reflejan en la encuesta contradicen los comportamientos mayoritarios de los españoles. Mientras que, en la actualidad, son una exigua minoría las parejas que tienen hijos sin estar casados, a diferencia de lo que ocurre en la mayoría de los países europeos, los entrevistados que dicen que es importante o muy importante casarse cuando se quieren tener hijos son menos que los que lo consideran poco o nada importante. En cierto modo, podemos considerar estas manifestaciones como reflejo de las actitudes que se van extendiendo por la sociedad española aunque todavía los comportamientos no son coherentes con ellas.

CuAdro 3. Grado de importancia que se concede al matrimonio cuando se piensa en tener hijos

¿Diría usted que es muy importante, poco importante o nada importante casarse cuando se quiere tener hijos?

\begin{tabular}{|c|c|c|c|c|c|c|c|c|c|}
\hline & \multirow{2}{*}{ Total } & \multicolumn{3}{|c|}{ Hombres Edades } & \multicolumn{4}{|c|}{ Mujeres Edades } \\
\cline { 3 - 10 } & muestra & Total & $18-25$ & $26-45$ & $46-65$ & Total & $18-25$ & $26-45$ & $46-65$ \\
\hline $\begin{array}{c}\text { Muy importante, } \\
\text { Importante }\end{array}$ & 44 & 48 & 36.5 & 42 & 64 & 40 & 32 & 31 & 57 \\
\hline $\begin{array}{c}\text { Poco importante, } \\
\text { Nada importante }\end{array}$ & 56 & 52 & 63.5 & 58 & 36 & 60 & 68 & 69 & 43 \\
\hline
\end{tabular}

Fuente: Encuesta propia «Flexibilidad, elección y estilos de vida familiar» 2000.

Consideramos que estas opiniones reflejan cambios importantes dada su contradicción con valores muy arraigados tradicionalmente en la sociedad española, como son los de la vinculación entre matrimonio y reproducción. Estos cambios son relativamente recientes, como reflejan las 
diferencias que advertimos entre unos grupos de edad y otros. Curiosamente, son las mujeres menores de 45 años el grupo que manifiesta conceder menos importancia al hecho de contraer matrimonio si se piensa en tener hijos.

Comparando con estudios anteriores también se confirma que en los últimos años han cambiado fuertemente las opiniones en esta materia. Según una encuesta de 1990 eran un 67\% los que daban importancia o mucha importancia a casarse antes de tener hijos y solo un $32 \%$ lo consideraban poco o nada importante (Alberdi, Flaquer e Iglesias 1994).

La comprensión que manifiestan los entrevistados de la convivencia sin matrimonio nos parece indicio de una relativa desinstitucionalización de la familia. Un $48 \%$ de los entrevistados dice que no viviría en pareja sin casarse personalmente, pero que comprende que otros lo hagan. $Y$ un $46 \%$ dice incluso que podría verse personalmente en esa situación. Entre hombres y mujeres hay una cierta diferencia que indica que las mujeres en mayor medida reflejan una comprensión desde el exterior de esta situación, mientras que los hombres en mayor medida muestran una cierta identificación personal. Entre los casados y no casados y según posiciones ideológicas de izquierda o derecha en las que se sitúan los individuos también se advierten diferencias en los niveles de tolerancia e identificación respecto de la convivencia sin matrimonio manifestándose más proclives, como era de esperar, los solteros y los que se sitúan políticamente más a la izquierda. (Cuadro 4)

Sin embargo, es la edad la variable que más discrimina en cuanto a las opiniones acerca de la convivencia sin matrimonio. Entre los más jóvenes aumenta enormemente las posiciones de tolerancia e identificación mientras que se reducen de forma significativa entre los mayores de 45 años.

CUADRo 4. Opiniones acerca de la convivencia sin matrimonio

\begin{tabular}{|l|c|c|c|c|}
\hline \multirow{2}{*}{ \% de los que dan la respuesta: } & \multirow{2}{*}{$\begin{array}{c}\text { Total } \\
\text { Muestra }\end{array}$} & \multicolumn{3}{|c|}{ Edades } \\
\cline { 3 - 5 } & & $18-25$ & $26-45$ & $46-65$ \\
\hline $\begin{array}{l}\text { Yo nunca lo haría y desapruebo totalmente } \\
\text { que la gente lo haga }\end{array}$ & $\mathbf{6}$ & 0.5 & 3 & 13 \\
\hline $\begin{array}{l}\text { Yo, personalmente, nunca lo haría pero } \\
\text { acepto y comprendo que otros lo hagan }\end{array}$ & $\mathbf{4 8}$ & 32 & 43 & 64.5 \\
\hline $\begin{array}{l}\text { Yo puedo imaginarme a mi mismo en esta } \\
\text { situación }\end{array}$ & $\mathbf{4 6}$ & 67.5 & 54 & 22.5 \\
\hline
\end{tabular}

Fuente: Encuesta propia «Flexibilidad, elección y estilos de vida familiar» 2000. 
Tenemos que analizar otras opiniones para entender porqué se casan los que lo hacen, dada esa enorme tolerancia, al menos como declaración, de la convivencia sin matrimonio. Para ello nos ayudan las explicaciones que dan los entrevistados acerca de las razones que consideran que influyen para casarse en lugar de vivir juntos. Las "presiones familiares» son las que más se señalan, un $66 \%$ de los entrevistados considera que las "presiones familiares» influyen mucho o bastante para que las parejas contraigan matrimonio en lugar de irse a vivir juntos. Esta es una opinión similar entre individuos de ambos sexos y de todas las edades. En segundo lugar, se señala el tener hijos o el pensar en tenerlos (60\% suman los que dicen que influye mucho o bastante) y razones prácticas, de comodidad para evitarse problemas de todo tipo (60\% entre mucho y bastante). Y a continuación, en mucha menor medida, se señalan como razones las creencias religiosas (51\% entre mucho y bastante), la estabilidad de la pareja (48\% entre mucho y bastante) y las ventajas económicas (48\% entre mucho y bastante).

Es interesante señalar que las declaraciones acerca de la existencia de presiones familiares, la razón más advertida por todos, es coherente con las diferentes actitudes ante el matrimonio y la convivencia que tienen los mayores y menores de 45 años y que explicaría, en cierta forma, la existencia de presiones por parte de las generaciones mayores sobre los jóvenes para empujarlos a contraer matrimonio.

\section{Estabilidad del matrimonio}

Queríamos saber en qué tipo de matrimonio se está pensando cuando se habla de parejas bien avenidas; y cuales son las variables a las que se da mayor importancia para que un matrimonio funcione.

De forma similar a como se ha visto en estudios anteriores, los aspectos que más se valoran para el éxito del matrimonio son los de la «fidelidad», la «comprensión y tolerancia» y el «respeto y cariño mutuos». A estas tres variables es a las que más frecuentemente se las señala como índices de estabilidad y armonía matrimonial. En un segundo lugar, aparecen las «relaciones sexuales satisfactorias» como factor de éxito en el matrimonio. Mucha menor consideración reciben las cuestiones ideológicas como «compartir las creencias religiosas» 0 «compartir las afinidades políticas» que aparecen como las menos señaladas. En una posición intermedia se señala la importancia de ciertas cuestiones materiales como el «tener ingresos adecuados», el tener «una vivienda en condiciones» y el 


\section{Cambios en los roles familiares y domésticos}

«vivir independientes de las familias políticas». El «tener hijos», el «tener gustos o intereses comunes» y el «compartir las tareas domésticas» reciben una importancia menor. (Cuadro 5)

CUADRO 5. Factores que se señalan como muy importantes o bastante importantes para que un matrimonio vaya bien. España 1981, 1990, 2000

Porcentaje de encuestados que da importancia a cada factor

\begin{tabular}{|c|c|c|c|}
\hline & \multicolumn{3}{|c|}{ Años } \\
\cline { 2 - 4 } & 1981 & 1990 & 2000 \\
\hline Fidelidad & 96 & 96 & 99.5 \\
\hline Respeto y cariño mutuo & 96 & 97 & 99 \\
\hline Comprensión y tolerancia & 95 & 5 & 99 \\
\hline Relación sexual satisfactoria & 90 & 90 & 95 \\
\hline Tener gustos e intereses comunes & 88 & 85 & 86 \\
\hline Tener hijos & 94 & 80 & 82 \\
\hline Ingresos adecuados & 85 & 90 & 89 \\
\hline Buenas condiciones de vivienda & 79 & 76 & 85 \\
\hline Compartir tareas domésticas & 61 & 64 & 83 \\
\hline No vivir con la familia política & 70 & 70 & 85 \\
\hline Pertenecer al mismo nivel social & 69 & 63 & 54 \\
\hline Compartir las creencias religiosas & 66 & 54 & 47 \\
\hline Compartir las posiciones políticas & 50 & 35 & 30 \\
\hline Que la mujer trabaje fuera de casa & $*$ & 43 & 52 \\
\hline
\end{tabular}

Fuente: Encuestas CIS 1981 y 1990. Referencia en Cruz Cantero y Cobo. Las mujeres españolas, lo privado y lo público, CIS 1991 y Encuesta propia «Flexibilidad, elección y estilos de vida familiar», 2000 .

Cuando comparamos las respuestas a nuestra encuesta con otras encuestas realizadas hace 10 y 20 años advertimos algunos cambios. Los encuestados señalan los mismos factores fundamentales que dan éxito y estabilidad a un matrimonio: la comprensión, el cariño, la fidelidad y las relaciones sexuales satisfactorias. Sin embargo, se aprecian cambios con relación a otros factores que no son los más fundamentales pero que pa- 
recen también tener influencia en que un matrimonio vaya bien. En estos años ha aumentado la importancia de compartir las tareas domésticas y el que las mujeres también trabajen fuera de casa como factores positivos para apoyar un matrimonio. Por el contrario ha disminuido la importancia de tener hijos como apoyo del matrimonio.

En todas estas cuestiones las opiniones expresadas por los hombres y las mujeres de todos los grupos de edad son similares, salvo en la cuestión de compartir las tareas domésticas, aspecto que cobra mayor importancia entre los jóvenes y algo más entre las mujeres que los hombres, en cuanto se valora como algo que hace que un matrimonio vaya bien. (Cuadro 6).

CUADRo 6. El compartir las tareas domésticas como factor muy importante para que un matrimonio vaya bien

Porcentajes de los que contestan que el compartir las tareas domésticas es una aspecto muy importante para que un matrimonio vaya bien

\begin{tabular}{|c|c|c|c|c|c|c|c|c|c|}
\hline & \multirow{2}{*}{$\begin{array}{c}\text { Total } \\
\text { muestra }\end{array}$} & \multicolumn{4}{|c|}{ Hombres Edades } & \multicolumn{4}{c|}{ Mujeres Edades } \\
\cline { 3 - 9 } & & Total & $18-25$ & $26-45$ & $46-65$ & Total & $18-25$ & $26-45$ & $46-65$ \\
\hline $\begin{array}{c}\text { Compartir las } \\
\text { tareas } \\
\text { domésticas es } \\
\text { muy importante }\end{array}$ & 38 & 34 & 36.5 & 41 & 25 & 42 & 48.5 & 45 & 34 \\
\hline
\end{tabular}

Fuente: Encuesta propia «Flexibilidad, elección y estilos de vida familiar» 2000.

Según algunos estudios, son las parejas menos convencionales, las de aquellos que conviven sin estar casados, los que dan mayor importancia a esta cuestión de participar los dos en el mundo laboral y compartir el trabajo doméstico (Alabart, Cabré et al. 1988). Entre los entrevistados que dicen vivir en pareja se confirman esta hipótesis pues ellos son los que señalan en mayor medida la importancia de compartir las tareas domésticas para que un matrimonio vaya bien. Un 56\% lo consideran muy importante, y un 33\% importante. Para este grupo, la fidelidad y el respecto y cariño mutuo adquieren sus valores máximos, no solo porque son los primeros sino también porque son más elevados que los de ningún 
otro grupo; después sitúan en importancia el tener relaciones sexuales satisfactorias y a continuación señalan este aspecto de compartir los trabajos domésticos. Quizás, en estas repuestas, se estén sumando los factores edad y estilos de vida a la vez que la mayor frecuencia de convivencia en pareja entre las generaciones más jóvenes.

Otro indicio a través del cual se advierte que está cambiando el valor de la institución matrimonial es en la pérdida de importancia del matrimonio respecto a la reproducción y en como se considera que buena parte de los jóvenes contraen matrimonio debido a presiones familiares o por razones prácticas. Sin embargo, a pesar de estas opiniones, ya sea por unas u otras razones, en nuestro país una mayoría de las parejas que tienen un proyecto de vida en común estable contraen matrimonio. Habría que saber si esto se debe mayormente a presiones externas o se debe a valores intrínsecos del matrimonio que todavía tienen vigencia; Y qué importancia tiene el matrimonio con relación a otros logros de la vida de estas parejas jóvenes.

\section{Valor del matrimonio}

Para contestar estas preguntas hemos tratado de comparar una serie de valores fundamentales, como tener trabajo o tener un hijo, con el valor que el matrimonio ofrece en cuanto al logro de una vida completa. Mayoritariamente se piensa que tanto las mujeres como los hombres pueden tener una vida completa sin casarse. En mucha mayor medida se considera necesario tener un trabajo y tener un hijo para «que pueda considerarse que una mujer tiene una vida completa» que el hecho de casarse. No es posible que una mujer tenga una vida completa sin tener un trabajo, dice un 38\%; no es posible sin tener un hijo, dice un $44 \%$; y no es posible sin tener una pareja estable, dice un $49 \%$.

Es decir, sin tener pareja no es posible considerar que se tiene todo pero, sin embargo, del matrimonio se puede prescindir, un $73 \%$ cree que «puede considerarse que un hombre tiene una vida completa sin casarse» y un $70 \%$ piensa lo mismo si se trata de una mujer. Esta es una opinión que disminuye con la edad, que disminuye más entre los hombres que entre las mujeres y entre las personas políticamente más conservadoras. (Cuadro 7). 
CUADRo 7. Aspectos necesarios para alcanzar una vida completa, en el caso de una mujer y en el caso de un hombre

Porcentajes de quienes afirman que se puede tener una vida completa en cada caso

\begin{tabular}{|l|c|c|c|c|}
\hline \multirow{2}{*}{$\begin{array}{c}\text { Una MUJER puede tener una } \\
\text { vida completa... }\end{array}$} & \multirow{2}{*}{$\begin{array}{c}\text { Total } \\
\text { muestra }\end{array}$} & \multicolumn{3}{|c|}{ Edades } \\
\cline { 3 - 5 } & & $18-25$ & $26-45$ & $46-65$ \\
\hline Sin casarse & 70 & 77 & 74 & 61 \\
\hline Sin tener una pareja estable & 49 & 50.5 & 50 & 47 \\
\hline Sin tener un trabajo & 38 & 30 & 36 & 46 \\
\hline Sin tener un hijo & 43 & 44 & 45 & 40 \\
\hline N.S/N.C & 1 & 0.5 & 1 & 1 \\
\hline
\end{tabular}

\begin{tabular}{|l|c|c|c|c|}
\hline \multirow{2}{*}{$\begin{array}{c}\text { Un HOMBRE puede tener una } \\
\text { vida completa... }\end{array}$} & \multirow{2}{*}{$\begin{array}{c}\text { Total } \\
\text { muestra }\end{array}$} & \multicolumn{3}{|c|}{ Edades } \\
\cline { 3 - 5 } & & $18-25$ & $26-45$ & $46-65$ \\
\hline Sin casarse & 73 & 78 & 74 & 69 \\
\hline Sin tener una pareja estable & 50 & 49 & 51 & 48 \\
\hline Sin tener un trabajo & 29 & 27 & 29 & 32 \\
\hline Sin tener un hijo & 44 & 43 & 46 & 42 \\
\hline N.S/N.C & 1.5 & 1 & 1 & 2 \\
\hline
\end{tabular}

Fuente: Encuesta propia «Flexibilidad. elección y estilos de vida familiar» 2000

\section{La importancia de tener hijos}

Más de la mitad de los entrevistados considera que tener un hijo es algo fundamental en la vida tanto si hablamos de los hombres como si pensamos en las mujeres. Tener hijos no se considera razón suficiente para contraer matrimonio ni tampoco un aspecto necesario para que un matrimonio vaya bien, sigue siendo, en sí mismo, un proyecto de gran importancia vital que, para buena parte de la población, es necesario para alcanzar una vida plena.

Estas opiniones se hacen algo más reducidas entre las mujeres menores de 25 años y entre los que viven en pareja sin contraer matrimonio. Podemos decir que el valor de los hijos, dentro de la vida de los individuos, se sitúa en una posición de importancia, se afirma como necesario por parte de la mitad de la población y como no necesario por parte de la otra mitad. Después del valor del trabajo, visto como necesario para te- 


\section{Cambios en los roles familiares y domésticos}

ner una vida completa, para la mayoría de los entrevistados, el tener un hijo es el siguiente asunto en importancia; tanto si se trata de una mujer o de un hombre.

La mayoría de los encuestados declara que no es necesario casarse cuando se van a tener hijos pero a la vez manifiestan como opción preferible que los niños vivan con el padre y con la madre. Es decir, parece perder importancia la legalización o no de la pareja pero, sin embargo, si se da gran importancia, cuando hay hijos, a la configuración de la familia sobre la base de la pareja estable del padre y de la madre. En este sentido, podemos decir que se siguen, por lo menos en el ámbito de opinión, los caminos recorridos en la evolución de las familias en los países europeos en los que buena parte de los niños nacen actualmente de padres no casados, pero que son parejas que conviven de forma estable, es decir que configuran una familia en la que el padre y la madre están presentes. Otra cuestión es que la ruptura de estas familias, ya estén basadas en matrimonio o en convivencia de hecho, pueda suponer para los hijos el pasar a vivir en una nueva forma de hogar en el que solo esté presente el padre o la madre.

Las opiniones de nuestros entrevistados reflejan la preocupación, cada vez más presente en la mayoría de las sociedades europeas, acerca de la vida de los niños que se crían solamente con uno de sus progenitores. Las opiniones están muy divididas, son más los que no creen que «un niño puede crecer felizmente viviendo solamente con su padre o solamente con su madre» pero es también un porcentaje muy elevado (el $43 \%$ ), el de los que creen que esto si es posible. (Cuadro 8)

CUADRO 8. Opiniones acerca de la necesidad de convivir con ambos progenitores paraque un niño pueda crecer felizmente.

Por sexo y edad

\begin{tabular}{|c|c|c|c|c|c|c|c|c|}
\hline $\begin{array}{c}\text { Un niño puede crecer } \\
\text { felizmente viviendo } \\
\text { solamente con su } \\
\text { madre o padre \% }\end{array}$ & \multicolumn{4}{|c|}{ Hombres Edades } & \multicolumn{5}{c|}{ Mujeres Edades } \\
\cline { 2 - 10 } & Total & $18-25$ & $26-45$ & $46-65$ & Total & $18-25$ & $26-45$ & $46-65$ \\
\hline De acuerdo & 41 & 47 & 44 & 32 & 44 & 59 & 49 & 29 \\
\hline En desacuerdo & 50 & 44 & 45 & 62 & 49 & 32 & 43 & 66 \\
\hline N.S/ N.C & 9 & 9 & 11 & 6 & 7 & 9 & 8 & 5 \\
\hline
\end{tabular}

Fuente: Encuesta propia «Flexibilidad, elección y estilos de vida familiar» 2000. 
La edad y el género diferencian las opiniones respecto de esta cuestión siendo las mujeres, y sobre todo las más jóvenes, las que creen en mayor medida que es posible crecer felizmente viviendo solo con la madre o solo con el padre. Podríamos interpretar que la posibilidad del divorcio, y la mayor frecuencia con que los hijos pasan a vivir con las madres después de la ruptura, inclinan a las mujeres en mayor medida a ver los aspectos positivos de esta situación.

Otro aspecto a destacar es como los hijos, aun cuando se consideran algo fundamental e importantísimo, han dejado de ser la justificación y el fin del matrimonio. Se han diversificado y se han flexibilizado las formas y las razones de tenerlos. El tener hijos se ha convertido en una opción y ha dejado de ser una obligación. Como consecuencia de ello la fecundidad se ha reducido enormemente en España en las dos últimas décadas y, acorde con esta evolución, también se ha reducido el «número ideal de hijos», es decir el número de hijos que la población, tengan o no tengan hijos, cree que el más adecuado para una familia. (Cuadro 9).

CuADro 9. Evolución del número ideal de hijos. España 1989-2000

Media de hijos que resulta de preguntar a todos los encuestados acerca de cual consideran que es el numero ideal de hijos para una familia

\begin{tabular}{|c|c|c|c|c|c|c|}
\hline & \multicolumn{7}{|c|}{ Años } \\
\cline { 2 - 7 } & $\mathbf{1 9 8 9}$ & $\mathbf{1 9 9 0}$ & $\mathbf{1 9 9 1}$ & $\mathbf{1 9 9 4}$ & $\mathbf{1 9 9 8}$ & $\mathbf{2 0 0 0}$ \\
\hline Media hijos & 2,59 & 2,5 & 2,49 & 2,31 & 2,34 & 2,15 \\
\hline
\end{tabular}

Fuente; Estudio CIS 1841 (1989), CIRES Encuesta matrimonios y parejas (1990), Estudio CIS 1968(1991), Estudio CIS 2113 (1994), Estudio CIS 2283 (1998), Encuesta propia «Flexibilidad, elección y Estilos de vida familiar» 2000.

Entre nuestros entrevistados domina, de acuerdo con la mayoría de las encuestas recientes, la preferencia por tener dos hijos. Un $69 \%$ de los entrevistados señalan esta cifra como el número ideal. Sin embargo, la preferencia de dos hijos por pareja está en contradicción con la realidad actual de la sociedad española en la que la media alcanza el 1,3 de hijos por mujer, y con la enorme cantidad de hombres y mujeres jóvenes que declaran, en estudios de tipo cualitativo, que no quieren tener más que un hijo.

El concepto de número ideal de hijos es un concepto, una opinión que no compromete personalmente, y que refleja la idea acerca de los hijos que los entrevistados piensan que sería mejor tener, en una situación 


\section{Cambios en los roles familiares y domésticos}

ideal de vida sin inseguridad económica y sin la presión de los problemas concretos. El ideal dominante es el de tener una pareja de hijos, a ser posible una hija y un hijo; ideal que tiene una persistencia grande en la sociedad española en los últimos años. La variación que nos interesa tener en cuenta es el declive de los partidarios de tener tres o más hijos y el aumento de los que piensan que lo ideal es tener uno solamente.

Esta evolución en el tiempo también se refleja en las opiniones de los distintos grupos de edad, siendo entre los hombres y mujeres mayores de 45 años entre los que se encuentra un mayor número de partidarios de las familias numerosas. Un $27 \%$ de los hombres y un $33 \%$ de las mujeres mayores de 45 años consideran como cifra ideal tener tres o más hijos.

Entre los entrevistados menores de 25 años, solo el $24 \%$ cifran el número ideal de hijos en tres, y entre los que tienen de 25 a 45 años descienden a 19 los que consideran como «ideal» tener lo que ahora se considera familia numerosa, es decir tres hijos. De modo que, a través de estos datos, advertimos que los españoles desean tener menos hijos que en el pasado y que, a la vez, tienen menos hijos de los que, idealmente, querrían tener.

Entre las razones que se señalan para explicar que la gente tenga menos hijos de los que en realidad desearía tener dominan las de carácter material, siendo las razones económicas las más señaladas por todos los entrevistados sin diferencias por edad, género, nivel de instrucción, situación familiar o posicionamiento ideológico. Un $83 \%$ de los preguntados señalan las razones económicas como causa para tener menos hijos y en consecuencia señalan las ayudas a las familias con hijos y las deducciones de impuestos como las medidas que serian más eficaces para tener más hijos.

De todas maneras, estas declaraciones globales encubren muchas razones diferentes, y todas pueden tener una traducción económica. Para entenderlas hay que relativizarlas y situarlas en el contexto personal de cada individuo en el que las dificultades económicas tiene significados muy diferentes, ya que no se produce, en el ámbito general, una correlación entre el bienestar económico de los individuos y el aumento del número de sus hijos. Por supuesto que los ingresos económicos, la estabilidad de estos ingresos, las condiciones de la vivienda y la multitud de factores que se engloban en esos términos de «condiciones económicas», van a influir en la decisión de tener hijos una vez que se ha pasado históricamente de la incapacidad de controlar la reproducción a una situación de libertad y voluntariedad de la misma.

En este sentido de entender las razones por las que las gentes deciden o evitan tener hijos, tenemos que señalar que una aproximación de 
carácter cuantitativo es menos rica en posibles matices que los estudios previos de carácter cualitativo que hemos realizado, en los que se ponían de manifiesto la diversidad de razones que los individuos tienen para tener o no tener hijos y para reducir el número de los mismos. (Cuadro 10)

Por supuesto que las razones principalmente señaladas son las económicas, pero es interesante advertir la importancia con que se señalan otro tipo de razones que ponen de relieve los cambios de estilo de vida que conlleva la paternidad y la maternidad. Las «cargas que los hijos implican» y el que «la mujer trabaje fuera de casa» aparecen como razones de peso para explicar que no se tengan tantos hijos como se desean. Es en estos aspectos en los que podemos advertir un cambio en la sociedad española, que explica, mejor que las solas variables económicas, el declive de la fecundidad. Nunca se ha vivido en la historia de España en unas condiciones económicas más favorables que ahora y sin embargo asistimos a un declive importante de la fecundidad. Por supuesto que todavía es pronto para comparaciones entre niveles de vida y fecundidad dado que el logro de la voluntariedad en la reproducción es algo recientemente alcanzado en la sociedad española y las altas tasas de fecundidad de los años sesenta y primeros setenta se produjeron en una situación de limitadas posibilidades de controlar la natalidad.

CUADRo 10. Razones para no tener hijos

¿Cuales cree que son las razones por las que algunas parejas no quieren tener hijos o tienen menos de los que en realidad desean?

\begin{tabular}{|l|c|c|c|c|}
\hline & \multicolumn{4}{|c|}{ Edades } \\
\cline { 2 - 5 } & TOTAL & $18-25$ & $26-45$ & $46-65$ \\
\hline Razones económicas & 83 & 85 & 84 & 80 \\
\hline Las cargas que implican los hijos & 28 & 32 & 27 & 26 \\
\hline Que la mujer trabaja fuera de casa & 22 & 18.5 & 22 & 24 \\
\hline Pesimismo ante la situación económica y social & 17 & 17 & 17 & 16 \\
\hline Tener una vivienda pequeña & 5 & 5 & 4 & 5 \\
\hline Problemas de salud de los padres & 1 & 1 & 1 & 1 \\
\hline $\begin{array}{l}\text { Miedo a que el hijo nazca con problemas de } \\
\text { salud }\end{array}$ & 1 & 0.5 & 2 & 1 \\
\hline
\end{tabular}

Fuente: Encuesta propia «Flexibilidad, elección y estilos de vida familiar» 2000

El trabajo remunerado de la mujer se señala en un $22 \%$ de los casos como una de las razones para tener menos hijos; hay que señalar que lo 
indican en mayor medida las mujeres mayores de 25 años, es decir aquellas que se enfrentan realmente con la decisión de tener hijos y con la responsabilidad de cuidarlos. Las «cargas que implican los hijos» se señalan como razón en un $28 \%$ de los casos y esta opinión aumenta entre los menores de 25 años, tanto hombres como mujeres. Quizás se refleje con ello ese miedo a perder libertad y tiempo libre que indican numerosos estudios realizados entre jóvenes como una de las dificultades mayores que se oponen a la decisión de tener hijos.

Respecto de cuales serian las ayudas más eficaces, después de las ayudas de tipo económico, aparece con una gran importancia el «promover el trabajo a tiempo parcial de las mujeres con hijos». Un 18,5\% lo señala en primer lugar y un $29 \%$ en segundo lugar, como medida eficaz. Son sobre todo las mujeres las que consideran el trabajo a tiempo parcial como forma de apoyar el tener hijos; incluso a las mujeres de entre $25 \mathrm{y}$ 45 años les parece una medida más eficaz que las deducciones en impuestos. La ampliación de los permisos de maternidad se señala en mucha menor medida, un $5 \%$ lo señala en primer lugar y un $12 \%$ en segundo lugar, y no aparecen las mujeres como especialmente partidarias de esta medida. En general estos datos coinciden con las percepciones encontradas en los trabajos cualitativos anteriores; los permisos de maternidad, tal y como están actualmente, no benefician a las madres trabajadoras tanto como deberían, quizás porque solo alcanzan a beneficiar a las trabajadoras con contrato indefinido y porque provocan, paralelamente a la posible ayuda que aportan, un efecto pernicioso por las reacciones negativas que suscitan entre los empresarios.

El aumento del número de guarderías se señala como posible medida para fomentar que la gente tenga hijos en una proporción muy reducida, $3 \%$ en primero y $8 \%$ en segundo lugar, siendo las personas con estudios superiores, las mujeres entre 25 y 45 años y los hombres entre 25 y 65 años los que más señalan esto como medida eficaz.

\section{La actividad femenina}

La incorporación de las mujeres al trabajo remunerado es uno de los cambios sociales que más ha influido en la transformación de los roles y las relaciones familiares. El aumento del empleo femenino en España, pese a que ofrece una de las cifras más reducidas de los países europeos, ha supuesto una transformación de las familias españolas y es de prever que esta tendencia se afiance, dada la diferencia de actividad femenina 
por grupos de edad. Las mujeres jóvenes tienen tasas de actividad mucho más elevadas que las mujeres mayores de 40 años y ello influye de forma creciente en las nuevas familias.

La idea de cómo deben ser los matrimonios, con relación a las actividades de hombres y de mujeres y en cuanto a como se obtengan los recursos económicos para la familia, ha cambiado mucho. Un $88 \%$ de los entrevistados dice estar de acuerdo en que «tanto el marido como la mujer deben contribuir a los ingresos del hogar». Es cierto que esta afirmación esta de acuerdo con las responsabilidades que el Código Civil desde 1981 atribuye a los hombres y a las mujeres en el seno del matrimonio y de la familia, pero no deja de llamar la atención una unanimidad tan alta en una sociedad en la que mayoritariamente las mujeres casadas no trabajan fuera del hogar y en la que las tasas de paro femenino se elevan hasta el doble de las tasas de paro masculino.

En el conjunto de nuestra muestra, son activas un $45 \%$ de las mujeres pero la actividad alcanza a un $58 \%$ para las que tienen entre 25 y 45 años. Además, entre las mujeres de esas edades que dicen que no trabajan, un 77,5 declaran que lamentan no tener empleo. Es interesante señalar que al preguntar las mismas cuestiones a los hombres que están casados con mujeres que no trabajan no parece que, según ellos, sus mujeres lamenten, en tanta medida, el no ser activas. Solo un $47 \%$ de estos maridos creen que sus mujeres lamentan no tener un empleo.

Las razones principales que señalan las mujeres como causa de no tener empleo remunerado, reflejan que esta no es una elección voluntaria; en primer lugar señalan la falta de tiempo debido a las responsabilidades familiares y en segundo lugar la escasez de empleo. Solo un $27 \%$ de estas mujeres que no trabajan dice que es por propia decisión. En este aspecto también se advierte un contraste entre la sensibilidad que tienen las mujeres que no tienen empleo remunerado y la opinión que expresan los maridos de mujeres en igual situación. Las razones para no trabajar por un sueldo son, según ellos, de carácter algo más voluntario, siendo un $39 \%$ las que, en opinión de sus maridos, están en esta situación por decisión propia.

Las razones por las que las mujeres buscan un empleo remunerado son muchas, en primer lugar razones económicas, comunes a la mayoría de los trabajadores; pero también se señalan razones de índole más personal, que se relacionan con las relaciones personales y la vida familiar. Por este orden, el tener independencia, el gusto por su profesión, el ganar un dinero extra, el relacionarse con otras personas, el sentido del deber y liberarse del encierro doméstico aparecen como ex- 


\section{Cambios en los roles familiares y domésticos}

plicaciones que las mujeres señalan como motivaciones de su actividad laboral. Las mujeres mayores de 45 años hablan más de relacionarse con otras personas y las menores de 45 años señalan en mayor medida el tener independencia como razón de su decisión de trabajar fuera de casa. (Cuadro 11).

CUADRo 11. Razones para trabajar que señalan las mujeres activas

\begin{tabular}{|l|c|c|c|c|}
\hline \multirow{2}{*}{$\begin{array}{c}\text { Razones que influyen en su decisión de } \\
\text { trabajar fuera de casa * } \\
\text { Porcentaje de cada respuesta }\end{array}$} & Total & \multicolumn{3}{|c|}{ Edades } \\
\cline { 3 - 5 } & & $\mathbf{1 8 - 2 5}$ & $\mathbf{2 6 - 4 5}$ & $\mathbf{4 6 - 6 5}$ \\
\hline Porque todas las personas deben trabajar & 18 & 17 & 20 & 21 \\
\hline Por necesidades económicas & 55 & 45 & 56 & 58 \\
\hline Por ganar dinero extra & 20 & 19 & 20 & 21 \\
\hline Por liberarme del trabajo doméstico & 11 & 2 & 12 & 15 \\
\hline Por relacionarme con otras personas & 20 & 14 & 19 & 25 \\
\hline Porque me gusta mi profesión & 30 & 29 & 30 & 31 \\
\hline Por tener independencia & 39 & 52 & 38 & 29 \\
\hline N.S/ N.C & 7 & 5 & 8 & 8 \\
\hline
\end{tabular}

Fuente: Encuesta propia «Flexibilidad, elección y estilos de vida familiar» 2000.

* Se podían dar hasta tres razones.

Los hombres entrevistados, cuya mujer trabaja fuera de casa, no señalan las razones de necesidad económica y de la búsqueda de la independencia tanto como las mujeres. Sin embargo, los hombres del grupo de edad entre 25 y 45 años, que coinciden en ser las cohortes de edad en que mayor número de mujeres son activas, dan como razones principales para explicar por qué sus mujeres trabajan las de las necesidades económicas (un 54\%), el gusto por su profesión (un 36\%) y la de tener independencia (un $33 \%$ ), coincidiendo con las declaraciones de las entrevistadas activas.

El trabajo remunerado es visto por las mujeres, crecientemente, como mecanismo de independencia y ello se refleja en las opiniones que, a lo largo de los últimos años, señalan el carácter imprescindible que cobra el 
trabajo remunerado para las mujeres. Un $38 \%$ cree que «una mujer puede tener una vida completa sin tener un trabajo", cifra que desciende hasta un $23 \%$ entre las mujeres de 18 a 25 años. Por otra parte, el $79 \%$ de nuestros entrevistados están de acuerdo en que «tener un empleo es imprescindible para poder ser independiente», cifra que llega al $94 \%$ entre las mujeres de 18 a 25 años.

Estos datos ponen de manifiesto el valor creciente que el trabajo remunerado tiene para las mujeres a las que, por otra parte, se les hace difícil compaginar su actividad y sus obligaciones familiares, no solo en tanto en cuanto son competitivos los tiempos dedicados a unas y otras actividades, sino también por la ideología que tradicionalmente ha adjudicado a las mujeres una serie de responsabilidades de cuidado y atención a los hijos y que aparece como un mecanismo de culpabilización de las mujeres trabajadoras que saben que, debido a su trabajo pueden dedicar menos horas a estar con sus hijos.

El valor que ha adquirido el trabajo remunerado para las mujeres es muy elevado, superando incluso al valor que tiene para los hombres. Según una encuesta de 1997, en los grupos de edad entre 25 y 45 años son más las mujeres que los hombres las que declaran que les gustaría tener un trabajo remunerado incluso aunque no necesitaran el dinero (CIS E.2235). La diferencia de género es pequeña, un $60 \%$ de las mujeres de 25 a 45 años afirman esto frente a un $59 \%$ de los hombres de la misma edad.

Otro aspecto importante de las actitudes ante el trabajo de las mujeres hace referencia a lo que esto pueda influir en los hijos. A pesar de la frecuente realidad de mujeres jóvenes que tienen hijos y a la vez trabajan, la idea de que las relaciones entre madre e hijo se deterioran por ello es una constante de la mentalidad tradicional que tiene todavía bastante vigencia. El que las relaciones con sus hijos de las madres trabajadoras pueden ser tan buenas como las de las madres que no trabajan fuera de casa es una idea que se va abriendo camino con dificultad. Siempre han existido madres que trabajaban por un sueldo; es más, muy frecuentemente, las mujeres pioneras en dedicarse a las actividades laborales han sido madres que lo han hecho para sacar adelante a sus hijos. Las madres solteras, las viudas y las separadas de todas las épocas han trabajado, sobre todo por sus hijos. A pesar de ello, todavía se ve la actividad femenina con recelo desde la perspectiva de la situación de los hijos. (Cuadro 12 y Cuadro 13). 


\section{Cambios en los roles familiares y domésticos}

CUADRO 12. Evolución de las opiniones acerca de la relación de las madres activas con sus hijos

\begin{tabular}{|l|c|c|}
\hline $\begin{array}{c}\text { «Una madre que trabaja puede tener con sus } \\
\text { hijos una relación tan cálida y segura como } \\
\text { una madre que no trabaja» }\end{array}$ & $\mathbf{1 9 9 4}$ & $\mathbf{2 0 0 0}$ \\
\hline De acuerdo/ Muy de acuerdo & 55 & 62 \\
\hline En desacuerdo/ Muy en desacuerdo & 39 & 38 \\
\hline N.S/N.C & 6 & 0 \\
\hline
\end{tabular}

Fuente: Estudio CIS 2494 (1994), Encuesta propia «Flexibilidad, elección y estilos de vida familiar» $(2000)$.

Las opiniones acerca del impacto que el trabajo de las madres puede tener en sus hijos han evolucionado; nos da idea de su evolución el ver como son muy diferentes entre unos grupos de edad y otros. Las mujeres se muestran más de acuerdo que los hombres en que «una madre que trabaja puede tener con sus hijos una relación tan cálida y segura como la que no trabaja» pero es sobre todo entre los hombres y las mujeres más jóvenes entre los que encontramos mayor nivel de acuerdo con esta opinión, $73 \%$ y $76 \%$ respectivamente.

CUADRO 13. Opiniones acerca de la relación de las madres activas con sus hijos, por grupos de edad

\begin{tabular}{|c|c|c|c|c|}
\hline \multirow{2}{*}{$\begin{array}{c}\text { \% de las entrevistadas que dicen } \\
\text { estar de acuerdo o muy de acuerdo } \\
\text { con esta afirmación }\end{array}$} & $\begin{array}{c}\text { Total } \\
\text { muestra }\end{array}$ & $18-25$ & $26-45$ & $46-65$ \\
\cline { 3 - 5 } & & 74.5 & 64 & 52 \\
$\begin{array}{c}\text { Una madre que trabaja puede tener una } \\
\text { relación tan cálida y segura como una } \\
\text { madre que no trabaja }\end{array}$ & 62 & 52 & 50 & 65 \\
\hline $\begin{array}{c}\text { A un niño en edad preescolar le puede } \\
\text { perjudicar que su madre trabaje }\end{array}$ & 53 & & & \\
\hline
\end{tabular}

Fuente: Encuesta propia «Flexibilidad, elección y estilos de vida familiar» 2000. 
La gente joven empieza a pensar de otra manera. Pero los miedos no desaparecen fácilmente y la idea de que el trabajo de la madre perjudica a los hijos pequeños es mayoritaria en el ámbito de la población, un 53\% está de acuerdo con ella. El nivel de acuerdo con esta idea es todavía más importante entre determinados grupos sociales. Un $65 \%$ de los mayores de 45 años están de acuerdo con ella, algo más en el caso de los hombres y algo menos en el de las mujeres. Los casados, los que tienen hijos y los que se sitúan políticamente más a la derecha, son los que muestran un mayor nivel de acuerdo con estas ideas.

\section{Contradicción entre expectativas y realidades}

Nos ha parecido importante analizar en esta investigación las preferencias de la población acerca del tipo de familia en que les gustaría vivir, para detectar hacia dónde se dirigen los deseos de los entrevistados en cuanto a los modelos de familia, y en qué medida ese ideal se corresponde con su propia realidad.

Concretamente en el punto relativo a cual sea el ideal de familia los datos del estudio muestran dónde están situadas las aspiraciones mayoritarias, en un modelo de obligaciones y responsabilidades iguales entre el hombre y la mujer, que en la práctica real está bastante lejano del modelo en que realmente viven los entrevistados y que predomina en la sociedad española.

Se plantearon como modelos tres tipos de distribución de roles en la familia:

a) Una familia en la que tanto el hombre como la mujer trabajen fuera de casa y se repartan las tareas del hogar y el cuidado de los hijos (si los hubiera)

b) Una familia en la que la mujer trabaje menos horas fuera de casa y por tanto se ocupe en mayor medida que el hombre de las tareas del hogar y del cuidado de los hijos (si los hubiera)

c) Una familia donde solo el hombre trabaje fuera de casa y sea la mujer la que se ocupe de las tareas del hogar y del cuidado de los hijos (si los hubiera).

Acerca de estos tres tipos de familia, según el reparto de roles en la pareja, se preguntaba a los entrevistados cual se acercaba más a su modelo ideal de familia y a continuación se les preguntaba cuál era, de hecho, el modelo que más se acercaba a su propia familia. (Cuadro 14 y 15). 


\section{Cambios en los roles familiares y domésticos}

CUADRO 14. Opiniones acerca del tipo de familia que se prefiere.

Por edad

\begin{tabular}{|c|c|c|c|c|}
\hline \multirow{2}{*}{$\begin{array}{l}\text { Porcentaje de los que dicen } \\
\text { preferir una familia en la que: }\end{array}$} & \multirow{2}{*}{$\begin{array}{c}\text { Total } \\
\text { muestra }\end{array}$} & \multicolumn{3}{|c|}{ Edades } \\
\hline & & $18-25$ & $26-45$ & $46-65$ \\
\hline $\begin{array}{l}\text { Tanto el hombre como la mujer } \\
\text { trabajan y se reparten las tareas del } \\
\text { hogar y el cuidado de los hijos }\end{array}$ & 60 & 71 & 65 & 46 \\
\hline $\begin{array}{l}\text { La mujer trabaja menos horas fuera de } \\
\text { casa y se ocupa más que el hombre de } \\
\text { las tareas domésticas y del cuidado de } \\
\text { los hijos }\end{array}$ & 22 & 19 & 21 & 24 \\
\hline $\begin{array}{l}\text { Solo el hombre trabaja fuera de casa y } \\
\text { es la mujer la que se ocupa de las } \\
\text { tareas domésticas y del cuidado de los } \\
\text { hijos }\end{array}$ & 17 & 8 & 12 & 29 \\
\hline N.S/N.C & 2 & 2 & 2 & 1 \\
\hline
\end{tabular}

Fuente; Encuesta propia «Flexibilidad, elección y estilos de vida familiar» 2000.

CuAdro 15. Tipo de familia en la que se vive.

Por edad

\begin{tabular}{|c|c|c|c|c|}
\hline \multirow{2}{*}{$\begin{array}{l}\text { Porcentaje de los que dicen vivir } \\
\text { en una familia en la que: }\end{array}$} & \multirow{2}{*}{$\begin{array}{c}\text { Total } \\
\text { muestra }\end{array}$} & \multicolumn{3}{|c|}{ Edades } \\
\hline & & $18-25$ & $26-45$ & $46-65$ \\
\hline $\begin{array}{l}\text { Tanto el hombre como la mujer } \\
\text { trabajan fuera del hogar y se reparten } \\
\text { las tareas domésticas y el cuidado de } \\
\text { los hijos }\end{array}$ & 29 & 34 & 38 & 14 \\
\hline $\begin{array}{l}\text { La mujer trabaja menos horas fuera } \\
\text { de casa y se ocupa más que el hombre } \\
\text { de las tareas domésticas y del cuidado } \\
\text { de los hijos }\end{array}$ & 13 & 19 & 12 & 10 \\
\hline $\begin{array}{l}\text { Solo el hombre trabaja fuera de casa y } \\
\text { es la mujer la que se ocupa de las } \\
\text { tareas domésticas y del cuidado de los } \\
\text { hijos }\end{array}$ & 41 & 44 & 37 & 46 \\
\hline Otros tipos & 16 & 2 & 13 & 30 \\
\hline
\end{tabular}

Fuente; Encuesta propia « Flexibilidad, elección y estilos de vida familiar» 2000. 
Los datos de la encuesta muestran que la mayoría de la población señala su preferencia por el tipo de matrimonio de similares roles de género, en la que ambos trabajan y se reparten las tareas domésticas y familiares por igual. Más de la mitad de los entrevistados, un $60 \%$, optan por esta alternativa. Sin embargo el modelo más frecuente en la realidad es el de la distribución de responsabilidades diferentes entre el hombre y la mujer. Un $42 \%$ de los entrevistados señala que en la realidad viven en ese otro tipo de familia, aquel en el que solo el hombre trabaja y por tanto es la mujer la que se responsabiliza de todo lo relacionado con el hogar y la familia. Este tipo de familia con separación de roles por género representa el modelo español de toda la vida, que está lentamente reduciéndose, y que solo sigue siendo el ideal para un tercio de los hombres mayores de 45 años y para un cuarto de los encuestados con menor nivel educativo.

Sin embargo la realidad de este modelo, que no es el preferido por los entrevistados, es todavía el más extendido en la sociedad española.

Parece que el camino hacia una mayor igualdad en el reparto de las responsabilidades familiares está trazado y nos atrevemos a afirmar que las opiniones de la población a este respecto suponen la no posibilidad de retorno a modelos tradicionales de familia o de convivencia. La evolución hacia el nuevo modelo igualitario ha sido muy fuerte. A lo largo de las últimas décadas han aumentado los que prefieren este tipo de familia y vemos en las encuestas realizadas en los años noventa que se manifiesta con claridad esa preferencia mayoritaria hacia una pareja con roles laborales y domésticos iguales para el hombre y la mujer. (Cuadro16)

CUADRo 16. Evolución de las actitudes ante el tipo de familia igualitario (1990-2000) Porcentaje de los que señalan como modelo ideal la familia igualitaria

\begin{tabular}{|c|c|c|}
\hline \multirow{2}{*}{ Tipo de familia } & \multicolumn{2}{|c|}{ Años } \\
\cline { 2 - 3 } & $\mathbf{1 9 9 5}$ & $\mathbf{2 0 0 0}$ \\
\hline $\begin{array}{c}\text { Una familia en la que tanto hombre como mujer } \\
\text { trabajan fuera de casa y se reparten las tareas del } \\
\text { hogar y el cuidado de los hijos }\end{array}$ & 62 & 59 \\
\hline
\end{tabular}

Fuente: CIS Estudios 2485(1990), 2493 (1995), Encuesta propia «Flexibilidad, elección y estilos de vida familiar» (2000).

Las respuestas obtenidas a estas preguntas muestran un panorama muy distante entre las preferencias de la población en cuanto al modelo 


\section{Cambios en los roles familiares y domésticos}

de familia o convivencia que les parece el ideal y la realidad de la convivencia familiar en la que viven. (Cuadro 17)

Debido a este contraste que se advierte entre la realidad y los deseos nos atrevemos a decir que el conseguir un cambio en el modelo de familia es un objetivo, una meta difícil de alcanzar y muy deseada. A pesar de las trabas que tienen las mujeres para situarse en el mundo del trabajo y las dificultades que tienen los hombres para asumir un modelo de convivencia basado en el compartir, muy distinto al que tienen interiorizado por su educación familiar y social, creemos que la sociedad española valora como superior una pareja donde existe un reparto de roles equilibrado por género.

Este espacio que separa las aspiraciones y la realidad es el que conforma la dinámica de la evolución social y el que marca los objetivos que impulsan al cambio. Son los modelos a los que se aspira, y los deseos que movilizan los comportamientos, pero no como un ideal genérico, sino como un objetivo que hay que alcanzar, y más aún tomando como referencia lo que se tiene y se pretende transformar.

CuADRo 17. Distancia entre el tipo de familia que la mayoría de personas prefieren y el porcentaje de personas que viven en este tipo de familia. Por edades

Porcentaje de encuestados que tipo ideal la familia en la que el hombre y la mujer trabajan fuera de casa y se reparten las tareas del hogar y el cuidado de los hijos si los hubiera, y porcentaje de encuestados que viven realmente en una familia así.

\begin{tabular}{|c|c|c|c|c|}
\hline \multirow{2}{*}{ Porcentaje de los que: } & \multicolumn{4}{|c|}{ Edades } \\
\cline { 2 - 5 } & Total & $18-25$ & $26-45$ & $46-65$ \\
\hline Les gustaría vivir en una familia igualitaria & 60 & 71 & 65 & 45 \\
\hline $\begin{array}{c}\text { Los que viven realmente en una familia } \\
\text { igualitaria }\end{array}$ & 29 & 34 & 40 & 14 \\
\hline
\end{tabular}

Distancia cuantitativa entre los que desean un tipo de familia igualitaria y los que viven en una familia igualitaria

\begin{tabular}{|l|l|l|l|}
\hline 31 & 37 & 25 & 31 \\
\hline
\end{tabular}

Fuente: Encuesta propia «Flexibilidad, elección y estilos de vida familiar» 2000.

A continuación se analizarán los datos de la encuesta que ponen de manifiesto este contraste entre las preferencias y la realidad familiar a la que se pertenece, diferenciando según las variables de sexo y edad. 
Salta a la vista que los jóvenes son los abanderados del modelo igualitario, y que precisamente en el grupo de 18 a 25 años es dónde mayor diferencia hay entre sus aspiraciones y la realidad en la que viven. Esto se debe probablemente a que en este tramo de edad muchos jóvenes siguen viviendo en la casa paterna dónde el modelo de familia es mayoritariamente el tradicional. Proyectan para su futura familia ideal unas aspiraciones alejadas de lo que constituye su familia de origen. Son los más abiertos al cambio, pero en su mayoría no se han tenido que enfrentar en primera persona a las dificultades de compatibilizar trabajo y familia. En este sentido, el grupo de edad de 26 a 45 años es el más rompedor de hecho, porque aunque su modelo ideal igualitario sea ligeramente menor, en la práctica están luchando por conseguir el modelo de familia igualitaria y son el grupo en el que este está más implantado. (Cuadro 18).

CUADRo 18. Tipos ideales de familia y modelos de familia real a los que se pertenece Porcentaje de entrevistados que prefieren cada tipo de familia ideal y porcentaje de entrevistados que pertenecen a cada tipo de familia

\begin{tabular}{|c|c|c|c|c|c|c|c|c|}
\hline \multirow{3}{*}{$\begin{array}{c}\text { Tipo de familia que se } \\
\text { prefiere }\end{array}$} & \multicolumn{4}{|c|}{ Hombres } & \multicolumn{4}{|c|}{ Mujeres } \\
\hline & \multirow{2}{*}{ Total } & \multicolumn{3}{|c|}{ Edades } & \multirow{2}{*}{ Total } & \multicolumn{3}{|c|}{ Edades } \\
\hline & & $18-25$ & $26-44$ & $44-65$ & & $18-25$ & $26-44$ & $44-65$ \\
\hline $\begin{array}{c}\text { Ambos trabajan y se } \\
\text { reparten las tareas } \\
\text { domésticas y cuidado de los } \\
\text { hijos }\end{array}$ & 57 & 69 & 65 & 39 & 62.5 & 73 & 64 & 53 \\
\hline $\begin{array}{l}\text { La mujer trabaja menos } \\
\text { horas y se ocupa más del } \\
\text { hogar e hijos }\end{array}$ & 21 & 19 & 19 & 25 & 22 & 19 & 23 & 23 \\
\hline $\begin{array}{l}\text { Sólo el hombre trabaja y la } \\
\text { mujer se ocupa de hogar e } \\
\text { hijos }\end{array}$ & 20 & 10 & 15 & 34 & 13.5 & 6 & 10 & 23 \\
\hline \multicolumn{9}{|l|}{$\begin{array}{c}\text { Tipo de familia al que se } \\
\text { pertenece }\end{array}$} \\
\hline $\begin{array}{c}\text { Ambos trabajan y se } \\
\text { reparten las tareas } \\
\text { domésticas y cuidado de los } \\
\text { hijos }\end{array}$ & 32 & 33 & 44 & 16 & 26 & 36 & 32 & 12 \\
\hline $\begin{array}{l}\text { La mujer trabaja menos } \\
\text { horas y se ocupa más del } \\
\text { hogar e hijos }\end{array}$ & 11 & 14 & 9 & 10 & 15 & 25 & 14 & 10 \\
\hline $\begin{array}{l}\text { Sólo el hombre trabaja y la } \\
\text { mujer se ocupa de hogar e } \\
\text { hijos }\end{array}$ & 37 & 51 & 31 & 35 & 46.5 & 37 & 43 & 57 \\
\hline
\end{tabular}

Fuente: Encuesta propia «Flexibilidad, elección y estilos de vida familiar» 2000. 


\section{Cambios en los roles familiares y domésticos}

La preferencia hacia una familia igualitaria es algo mayor entre las mujeres que entre los hombres: $62.5 \%$ lo desean frente a un 57\% de los hombres. Esta diferencia adquiere más relevancia cuando se compara la familia real en la que los encuestados dicen vivir en su propia casa; más hombres que mujeres dicen vivir en familia igualitaria y más mujeres dicen vivir en familia tradicional. Esto se puede explicar porque los encuestados sean muy optimistas a la hora de analizar su situación, mientras que las mujeres son más conscientes de la diferencia de hecho que les separa del ideal al que aspiran. Tal interpretación indicaría que las mujeres son más propensas a protagonizar el cambio y que a su vez están más concienciadas de las dificultades que ello plantea. (Cuadro 18).

Cabe destacar que las mujeres de 26-45 años optan más que las de 18 a 25 años por la alternativa de trabajar a media jornada. En otra pregunta de la encuesta, las mujeres de 26 a 45 años se decantaron en un $29 \%$ por promover la jornada a tiempo parcial para mujeres con hijos, mientras que los hombres de su misma edad sólo apoyaban esta medida en un 15\%. La posible explicación de por qué las mujeres de este grupo de edad se muestran partidarias de una jornada laboral reducida para ocuparse más de los hijos, puede ser que estas mujeres son las que tienen que hacerse cargo de hijos pequeños. El 57\% de estas mujeres trabajan fuera del hogar, y un $75 \%$ de ellas tiene hijos.

La variable de tener hijos es muy significativa. Vemos como el modelo ideal para los encuestados sin hijos es el igualitario, en un $67 \%$ de los casos, y desciende considerablemente cuando tienen hijos al 54.7\%. Asimismo su situación real cambia, y pasan de un 35\% que viven en un tipo de familia igualitaria, desde el punto de vista de trabajo exterior y trabajo doméstico, cuando no tienen hijos, a ser tan solo un $25 \%$ cuando tienen hijos.

La explicación podríamos darla por sentido común. La sobrecarga real a la que se enfrentan estas mujeres con hijos pequeños las obliga a hacer un reajuste ideológico y ceder a una opción intermedia. La jornada a tiempo parcial es la opción o salida que permite que cuiden de los hijos pequeños sin tener que renunciar a su trabajo. Por tanto son ellas las que desean amortiguar la situación cediendo a la opción «menos mala». Pero una vez más se produce el desfase entre aspiraciones y realidad cuando vemos que solo el $62 \%$ de las entrevistadas que desean una jornada reducida la consiguen. Esto se debe sobre todo a las dificultades que plantea el mercado laboral, y la rigidez contractual que existe en España respecto a la jornada a tiempo parcial y a las estrategias de flexibilización del tiempo de trabajo. 
Esta demanda por parte de las mujeres se constató en el estudio cualitativo previo a esta encuesta en el que apareció con gran énfasis la demanda que presentaban muchas mujeres con hijos pequeños de lograr una reducción de las jornadas laborales para poder conciliar trabajo y familia, con posterior posibilidad de reincorporarse plenamente al trabajo una vez que los hijos fueran lo suficientemente mayores.

Por otra parte, la posición ideológica también influye en estas preferencias de modelo familiar. Cuanto más a la izquierda política se sitúa el encuestado, más prefiere y más se encuentra en el modelo familiar igualitario.

Otra variable de interés es el nivel educativo, estrechamente ligado a la clase social. (Cuadro 19).

CuAdro 19. Distancia entre el tipo de familia ideal igualitario y el modelo de familia al que se pertenece, por nivel de estudios

Porcentaje de entrevistados que prefiere el tipo ideal igualitario (ambos trabajan y se reparten tareas del hogar y cuidado de los hijos) y porcentaje que afirma pertenecer a una familia de este tipo en la realidad.

\begin{tabular}{|c|c|c|c|c|}
\hline & \multirow{2}{*}{ Total } & \multicolumn{3}{|c|}{ Nivel de Estudios } \\
\cline { 3 - 5 } & & EGB y menos & Secundarios & Superiores \\
\hline $\begin{array}{c}\text { Tipo ideal de familia igualitaria que } \\
\text { se prefiere }\end{array}$ & 60 & 50 & 67 & 76 \\
\hline $\begin{array}{c}\text { Tipo de familia igualitaria a la que se } \\
\text { pertenece en realidad }\end{array}$ & 29 & 20 & 38 & 41 \\
\hline
\end{tabular}

Distancia entre la familia ideal que se prefiere y familia a la que se pertenece en realidad

\begin{tabular}{|l|l|l|l|}
\hline 31 & 30 & 29 & 35 \\
\hline
\end{tabular}

Fuente: Encuesta propia «Flexibilidad, elección y estilos de vida familiar» 2000.

Hay una notable diferencia en cuanto a los modelos familiares tanto en el plano de las preferencias como en el plano de las realidades de convivencia. Los entrevistados de mayor nivel educativo son los que más prefieren el modelo igualitario, un 76\%, y los que más lo viven en su propia familia, un $40 \%$, mientras que los entrevistados de nivel educativo de EGB y menos muestran en mucha menor medida, un 50\%, preferencia por la familia simétrica y aun mucho menos, un $20 \%$, viven realmente en este tipo de familia. Esta diferencia evidencia que el estrato social más favorecido culturalmente y socialmente es el que está protagonizando el 


\section{Cambios en los roles familiares y domésticos}

cambio. Esto tiene mucho que ver con que las mujeres con niveles de cualificación superior son precisamente las más proclives a trabajar fuera de casa, y las que tienen mayores oportunidades de obtener un puesto de trabajo lo suficientemente bueno como para igualarse con su pareja en estatus y en ingresos. Las mejores posibilidades laborales de la mujer dentro de una pareja es un elemento muy importante para el cambio de su posición en la familia.

Como conclusión, podemos decir que cada vez está más arraigado el modelo igualitario de roles y ello empuja al abandono de los modelos vividos en la familia de origen. Es un cambio en gran medida generacional, que presenta todavía resistencias culturales en cuanto que los hombres y la cultura empresarial no asumen plenamente las consecuencias del cambio en el sentido de la incorporación de los hombres a las tareas domésticas y familiares.

Hasta ahora, en esta cuestión del reparto de roles domésticos y laborales, las mujeres son las más desfavorecidas por su desventaja en el mercado de trabajo y por sus responsabilidades familiares escasamente compartidas. Muchas mujeres se encuentran pilladas en un círculo vicioso difícil de romper: su propia situación familiar les impide satisfacer unas expectativas laborales que, serian precisamente las que les permitirían cambiar su situación familiar hacia la igualdad.

Es decir, la discriminación laboral de las mujeres se debe a que son consideradas las responsables de su casa y de sus hijos, y ello actúa como obstáculo para que su trabajo sea equivalente al de sus parejas y para que los hombres asuman su parte correspondiente del trabajo doméstico y familiar. Como consecuencia de esta situación, el mayor desencadenante del cambio es el empuje de las mujeres que logran entrar en el mercado de trabajo, pues la igualdad doméstica se justifica en la medida en la que consigan igualarse a los hombres en el terreno laboral. Este avance se da en mayor medida en las mujeres más cualificadas, y por eso entre ellas hay menos distancia entre el tipo de familia en la que viven y el tipo de familia en la que desearían vivir.

A pesar de las dificultades y de los deseos insatisfechos que reflejan, cabe realizar una lectura optimista de los datos, ya que de éstos no se desprende una actitud conformista o resignada, sino una voluntad de cambio. Es más, se puede definir como una voluntad sentida desde dentro, acuñada en el propio caldo de cultivo de la insatisfacción, y no solo como producto de imitación de otros modelos familiares extranjeros. 